\title{
Generation of Large Flavor Mixing from Radiative Corrections
}

\author{
K. R. S. Balaji* \\ Institute of Mathematical Sciences, Chennai 600 113, India \\ Amol S. Dighe ${ }^{\dagger}$ \\ Theory Division, CERN, CH-1211 Geneva 23, Switzerland \\ R. N. Mohapatra \\ Department of Physics, University of Maryland, College Park, Maryland 20742 \\ M. K. Parida ${ }^{\S}$ \\ Department of Physics, North Eastern Hill University, Shillong 793022, India
}

(Received 11 February 2000)

\begin{abstract}
We provide a model independent criterion which would guarantee a large flavor mixing of two quasidegenerate Majorana neutrinos at the low scale, irrespective of the mixing at the high scale. We also show that such a situation is realizable for a phenomenologically interesting range of parameters of the weak scale theory. We further claim that for a similar condition to be implementable for the three generation case, the $C P$ parity of one of the neutrinos needs to be opposite to that of the others.
\end{abstract}

PACS numbers: $14.60 . \mathrm{Pq}, 11.30 . \mathrm{Hv}, 12.15 . \mathrm{Lk}$

I. Introduction. - The observed atmospheric and solar neutrino anomalies from the terrestrial experiments [1,2] seem to provide strong evidence in favor of neutrino flavor conversions, implying that neutrinos are massive and they mix among themselves. While the details are fuzzy at this stage, it is clear that the atmospheric neutrino data require a large mixing of $\nu_{\mu} \leftrightarrow \nu_{\tau}$ or $\nu_{\mu} \leftrightarrow \nu_{s}$, although the latter possibility is beginning to look less and less likely [3]. Regarding the solar neutrino anomaly, the mixing angle (between $\nu_{e}$ and $\nu_{\mu, \tau}$ or $\nu_{s}$ ) could be small, as in the small angle MSW scenario, or large, as in the large angle MSW or vacuum oscillation scenarios [2].

Since large mixing angles are involved in the possible solutions of both the anomalies, a great deal of theoretical work has gone in the understanding of the maximal mixing. There are two complementary approaches: (i) searching for scenarios and symmetries beyond the standard model, and (ii) establishing general model independent criteria which guarantee the stability at the weak scale of the masses and mixing pattern that emerge at the high scale. The second approach has the advantage that it may not only narrow down the search for new physics scenarios to a manageable level, but it may also throw light on the parameters of the theory at the high scale, and on the value of the high scale itself. In this paper, we present some model independent criteria for such theories, first focusing on the two flavor case and subsequently on the three flavor models.

If neutrinos contribute even a small fraction of the dark matter [4] of the universe, the oscillation observations imply a situation where at least two neutrinos (and possibly even three) are quasidegenerate in mass. If the neutrino mass hierarchy is inverted $\left(m_{3}<m_{1}, m_{2}\right.$ where $\left.\left|\Delta m_{32}^{2}\right| \gg\left|\Delta m_{21}^{2}\right|\right)$, the neutrinos $\nu_{1}$ and $\nu_{2}$ are necessar- ily quasidegenerate. The study of scenarios where two or even three neutrinos are nearly degenerate is therefore of crucial importance.

The two flavor quasidegenerate neutrino scenarios fall in two classes: the neutrino flavors in the degenerate limit can be (i) in the same $C P$ eigenstate or (ii) in opposite $C P$ eigenstates. It turns out that not only does one need to invoke different kinds of symmetries to understand the two cases, but the radiative corrections to the tree level degeneracy at the high scale can have very different implications for the two cases. For instance, it has been noted in [5-7] that in the case (ii), the radiative corrections (such as those through the RGE evolution from the seesaw to the weak scale) do not substantially affect the maximal mixing and quasidegeneracy predicted by the theory at a high scale.

In this analysis, we point out that in the case (i), an arbitrary mixing at the high scale can get "magnified" to a large mixing, and even possibly maximal mixing, at the low scale. We find this interesting because (a) it enables a model builder to avoid any fine tuning for the values of mixing angles at the high scale, and hence relaxes the constraints on the parameters of the high scale physics, (b) it brings a certain unity in the understanding of the quark and lepton mixings. This is arrived at by relating the radiative corrections and the degree of mass degeneracy $\left(\frac{\delta m}{m}\right)$, regardless of the mixing pattern at the high scale. In the context of specific models, this also leads to relationships between the degree of degeneracy, the value of the high scale, and the model parameters (e.g., $\tan \beta$ for the MSSM). We further extend the results to the three generation scenario and find that the constraint on $U_{e 3}$ from the CHOOZ experiment [8] indicates that the $C P$ parity of one of the neutrinos must be opposite to that of the others for our scheme to be implementable. 
Our paper is organized in the following form: in the next section, we derive our main result for the two flavor mixing. In section III, we present implications of the two flavor result for the case of the standard model and the MSSM. In section IV, we consider the extension to three generation case.

II. Basic formalism for two flavors. - Consider the mixing of two neutrinos. The $2 \times 2$ Majorana matrix in the mass basis is of the form

$$
\mathcal{M}_{D}=\left(\begin{array}{cc}
m_{1} & 0 \\
0 & m_{2}
\end{array}\right) .
$$

The unitary matrix which takes $\mathcal{M}_{D}$ to the flavor basis can be written as

$$
U=\left(\begin{array}{cc}
C_{\theta} & S_{\theta} \\
-S_{\theta} & C_{\theta}
\end{array}\right)\left(\begin{array}{cc}
1 & 0 \\
0 & e^{i \phi / 2}
\end{array}\right),
$$

where $\theta$ is the mixing angle and $\phi$ is the $C P$ phase. All the quantities are defined at the high scale $\Lambda$. The two neutrino flavors are related to the mass eigenstates in the conventional form

$$
\nu_{f}=U_{f i} \nu_{i}, \quad f=\alpha, \beta ; i=1,2 .
$$

We define the convention for the "numbering" of $\nu_{1}$ and $\nu_{2}$ as follows. Let $\nu_{\alpha}$ and $\nu_{\beta}$ be the $\mathrm{SU}(2)_{L}$ partners of the charged leptons $\ell_{\alpha}$ and $\ell_{\beta}$, respectively, such that $m_{\ell_{\alpha}}<m_{\ell_{\beta}}$. Then we define $\nu_{1}\left(\nu_{2}\right)$ as the state with a larger component of the flavor $\alpha(\beta)$ at the high scale. With this convention, $0 \leq \theta \leq \pi / 4$.

The mass matrix in the flavor basis can be written as

$$
\begin{aligned}
\mathcal{M}_{F} & =U^{*} \mathcal{M}_{D} U^{\dagger} \\
& =\left(\begin{array}{cc}
C_{\theta} & S_{\theta} \\
-S_{\theta} & C_{\theta}
\end{array}\right)\left(\begin{array}{cc}
m_{1} & 0 \\
0 & m_{2} e^{-i \phi}
\end{array}\right)\left(\begin{array}{cc}
C_{\theta} & -S_{\theta} \\
S_{\theta} & C_{\theta}
\end{array}\right) .
\end{aligned}
$$

Let us examine the situation when $\phi=0$, which corresponds to the case when the neutrinos $\nu_{1}$ and $\nu_{2}$ are in the same $C P$ eigenstate. Because of the presence of radiative corrections to $m_{1}$ and $m_{2}$, the matrix $\mathcal{M}_{F}$ gets modified to

$$
\mathcal{M}_{F} \rightarrow\left(\begin{array}{cc}
1+\delta_{\alpha} & 0 \\
0 & 1+\delta_{\beta}
\end{array}\right) \mathcal{M}_{F}\left(\begin{array}{cc}
1+\delta_{\alpha} & 0 \\
0 & 1+\delta_{\beta}
\end{array}\right)
$$

In the above, $\delta_{\alpha}$ and $\delta_{\beta}$ denote the corrections to the masses in the flavor basis. The above general structure for $\mathcal{M}_{F}$ has been motivated by the RGE structure for radiative corrections [9]. We define

$$
\epsilon \equiv 2\left(\delta_{\beta}-\delta_{\alpha}\right),
$$

which is the net difference in the radiative corrections for the masses of the two neutrino flavors.

The mixing angle $\bar{\theta}$ that now diagonalizes the matrix $\mathcal{M}_{F}$ at the low scale $\mu$ (after radiative corrections) can be related to the old mixing angle $\theta$ through the following expression:

$$
\tan 2 \bar{\theta}=\tan 2 \theta\left(1+\delta_{\alpha}+\delta_{\beta}\right) \frac{1}{\lambda},
$$

where

$$
\lambda \equiv \frac{\left(m_{2}-m_{1}\right) C_{2 \theta}+2 \delta_{\beta}\left(m_{1} S_{\theta}^{2}+m_{2} C_{\theta}^{2}\right)-2 \delta_{\alpha}\left(m_{1} C_{\theta}^{2}+m_{2} S_{\theta}^{2}\right)}{\left(m_{2}-m_{1}\right) C_{2 \theta}} .
$$

In the case of near degeneracy: $m_{1} \approx m_{2} \approx m$, we have

$$
\lambda=\frac{m \epsilon}{\left(m_{2}-m_{1}\right) C_{2 \theta}}+1,
$$

where $m$ is the common mass scale of the neutrinos. If

$$
|m \epsilon| \gg\left|\left(m_{2}-m_{1}\right) C_{2 \theta}\right|,
$$

then $\lambda \rightarrow \infty$ and we have $\tan 2 \bar{\theta} \rightarrow 0$. Under this condition, any mixing angle tends to zero after radiative corrections, i.e., a large mixing is unstable under radiative corrections. Note that this is true only for two neutrinos with the same $C P$ parity. If they had different $C P$ parities, i.e., $\phi=\pi$, quasidegeneracy would imply $\left|m_{1}\right| \approx\left|m_{2}\right| \approx m$, however $\left|m_{1}-m_{2}\right| \approx 2 m$. Then the radiative corrections (which are small) cannot give the inequality (10). In this case, $|m \epsilon| \ll\left|\left(m_{2}-m_{1}\right) C_{2 \theta}\right|$, so that $\lambda \approx 1$ and the mixing angle does not change much. The mixing at the high scale then remains stable. This reproduces the observations made in $[5,6]$ regarding the stability of the Maki-Nakagawa-Sakata (MNS) mixing matrix [10], when the mixing angle is close to $\pi / 4$. In addition, our analysis shows that the same conclusions remain valid for any arbitrary nonzero $\theta$ of the MNS matrix.
If

$$
\begin{aligned}
\left(m_{1}-m_{2}\right) C_{2 \theta}= & 2 \delta_{\beta}\left(m_{1} S_{\theta}^{2}+m_{2} C_{\theta}^{2}\right) \\
& -2 \delta_{\alpha}\left(m_{1} C_{\theta}^{2}+m_{2} S_{\theta}^{2}\right),
\end{aligned}
$$

then $\lambda=0$ or equivalently $\bar{\theta}=\pi / 4$; i.e., maximal mixing. Given the mass hierarchy of the charged leptons: $m_{l_{\alpha}} \ll m_{l_{\beta}}$, we expect $\left|\delta_{\alpha}\right| \ll\left|\delta_{\beta}\right|$, which reduces (11) to a simpler form:

$$
\epsilon=\frac{\delta m C_{2 \theta}}{\left(m_{1} S_{\theta}^{2}+m_{2} C_{\theta}^{2}\right)},
$$

where $\delta m \equiv m_{1}-m_{2}$. In the quasidegenerate case,

$$
\epsilon \approx \frac{\delta m}{m} C_{2 \theta} \text {. }
$$

The above expression can be translated in terms of the mass-squared difference (which is the quantity measured in the oscillation experiments) as

$$
\epsilon \approx \frac{\Delta m^{2}(\Lambda)}{2 m^{2}} C_{2 \theta}
$$

where $\Delta m^{2}(\Lambda)=m_{1}^{2}(\Lambda)-m_{2}^{2}(\Lambda)$. If the condition (14) is satisfied, the mixing at the scale $\mu$ tends to become 
maximal regardless of the value of the mixing at the scale $\Lambda$. Several points are worth emphasizing here.

(i) The above relation between $\epsilon$ and the neutrino parameters $\theta, m_{1}$, and $m_{2}$ is a model independent result and has profound implications for model building. For instance, it will relax the domain of parameters of the high scale theory compared to what was believed earlier for $\phi=0$.

(ii) From (14), the sign of $\epsilon$ must be the same as that of $\Delta m^{2}(\Lambda)$ for getting maximal mixing at the low scale. This preference is of a phenomenological importance since the sign of $\Delta m^{2}$ at low scales is measured by experiments: if the solar neutrino solution is MSW, the identity of the heavier neutrino is known, and the heavy/light nature of the third neutrino may be determined through the long baseline experiments [11] or the observations of a galactic supernova [12]. The model needs to be able to reproduce this sign from the values of the masses at the high scale through the RGE. The results of this paper can thus be used to discriminate between various models for a large flavor mixing.

(iii) The condition (14) is not to be mistaken for a fine tuning. Though maximal mixing at the low scale requires an exact equality (14), the condition can be slackened if we need only a large mixing. Indeed, the SK data [1] indicate $|\tan 2 \bar{\theta}|>2$ at $90 \%$ C.L. In Fig. 1, we show the range of $\epsilon$ that allows a large mixing at the scale $\mu$ as a function of the mixing angle at the scale $\Lambda$. The region enclosed within the "leaf" gives the range of $\epsilon$ which generates a large mixing $(|\tan 2 \bar{\theta}|>2)$. The value of the degree of degeneracy $\left|\frac{\delta m}{m}\right|$ chosen for the figure is 0.1 . From (13), changing this value would just change the scale of $\epsilon$ by a factor proportional to $\left|\frac{\delta m}{m}\right|$. The figure shows that a large mixing at the scale $\mu$ is indeed possible for a large range of neutrino parameters. The condition on the signs of $\epsilon$ and $\Delta m^{2}$ is also relaxed if the mixing at the high scale is already large.

III. Applications to the standard model and the MSSM. - In this section, we analyze the implications of (14) for the case of the standard model (SM) and MSSM to see whether it is satisfied for acceptable values of the model parameters. In the case of the SM, the value of $\epsilon_{\mathrm{SM}}$ from the RGE evolution is

$$
\epsilon_{\mathrm{SM}} \approx \frac{h_{\beta}^{2}}{32 \pi^{2}} \ln \left(\frac{\Lambda}{M_{Z}}\right),
$$

where $h_{\beta}$ corresponds to the Yukawa coupling of the heavier charged lepton. Equation (13) and the sign of $\epsilon_{S M}$ in (15) imply that for large flavor mixing to be generated in the SM through radiative corrections, we require $m_{1}>m_{2}$. In addition, from (14) and (15), the strength of $h_{\beta}$ needs to be

$$
h_{\beta}(\mathrm{SM}) \approx \sqrt{\frac{16 \pi^{2}\left|\Delta m^{2}(\Lambda)\right| C_{2 \theta}}{\ln \left(\frac{\Lambda}{M_{Z}}\right) m^{2}}} .
$$

This is a relation between $\Delta m^{2}(\Lambda)$ and the scale $\Lambda$ that needs to be obeyed. As an illustration, taking $\beta \equiv \mu$ (for

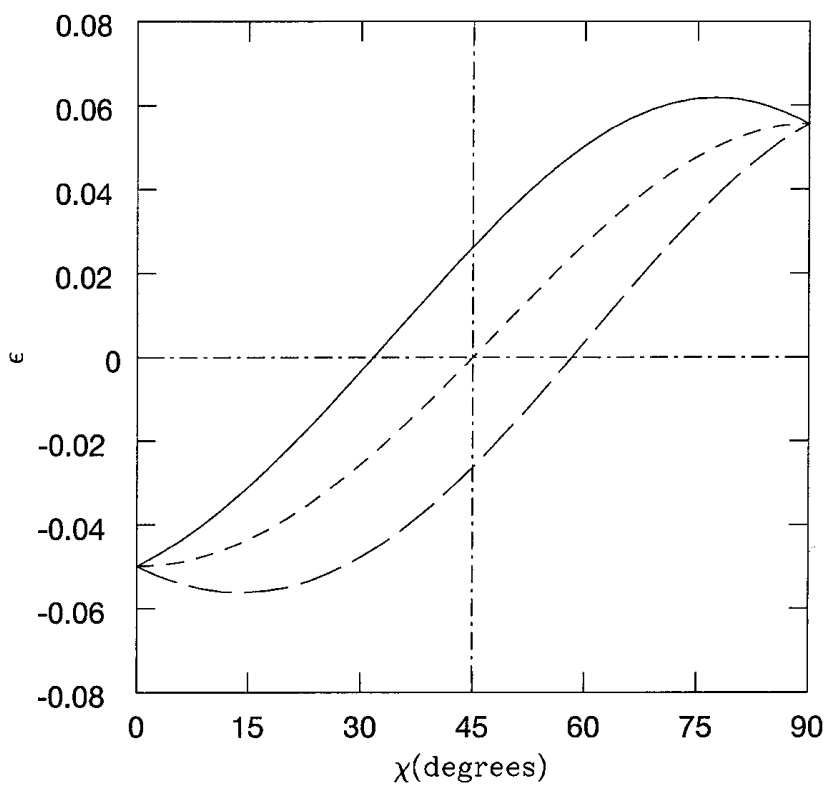

FIG. 1. The radiative correction $\epsilon$ that can generate a large mixing at the scale $\mu$ for $\left|\frac{\delta m}{m}\right|=0.1$. Here $\chi \equiv \theta$ for $m_{1}<$ $m_{2}$ and $\chi \equiv \frac{\pi}{2}-\theta$ for $m_{1}>m_{2}$. The central short-dashed line corresponds to maximal mixing $\left(\bar{\theta}=\frac{\pi}{4}\right)$. The solid (longdashed) line denotes $\tan 2 \bar{\theta}=2(-2)$.

$\nu_{e} \leftrightarrow \nu_{\mu}$, for example), with $h_{\mu} \approx 6 \times 10^{-4}$ and the high scale as $\Lambda \sim 10^{12} \mathrm{GeV}$, for degenerate neutrino mass of $m \sim 1 \mathrm{eV}$, we get $\left|\Delta m^{2}(\Lambda)\right| \approx 10^{-7} \mathrm{eV}^{2}$.

In the case of MSSM, we have

$$
\epsilon_{\mathrm{MSSM}} \approx-\frac{h_{\beta}^{2}}{16 \pi^{2}} \ln \left(\frac{\Lambda}{\mu}\right) .
$$

Equation (13) and the sign of $\epsilon_{\text {MSSM }}$ in (17) imply that we need $m_{1}<m_{2}$ for large flavor mixing to be generated through radiative corrections in the MSSM. In addition, from (14) and (17), the strength of $h_{\beta}$ has to be of the order of

$$
h_{\beta}(\mathrm{MSSM}) \approx \sqrt{\frac{8 \pi^{2}\left|\Delta m^{2}(\Lambda)\right| C_{2 \theta}}{\ln \left(\frac{\Lambda}{\mu}\right) m^{2}}} .
$$

Taking $\beta \equiv \tau$ (for $\nu_{\mu} \leftrightarrow \nu_{\tau}$ mixing, for example), and using

$$
h_{\tau} \approx \frac{m_{\tau}}{v \cos \beta},
$$

we get a relation between $\Lambda, \tan \beta$ and $m$, the common mass scale of the neutrinos. From (18) and (19), for given $m$ and $\Lambda$, we can infer the desirable value of $h_{\tau}$ and hence of $\tan \beta$. For example, for $m \sim 1 \mathrm{eV}$ and $\Lambda \sim 10^{12} \mathrm{GeV}$, taking $\left|\Delta m^{2}(\Lambda)\right| \approx 10^{-3} \mathrm{eV}^{2}$, we get $\tan \beta \approx 5$.

IV. Extension to three generations. - Let us now make a few comments on the possible extension to the case of three quasidegenerate Majorana neutrinos. If $m_{\alpha \beta}$ are the elements of the neutrino mass matrix in the flavor basis, then in the approximation of the decoupling of the third 


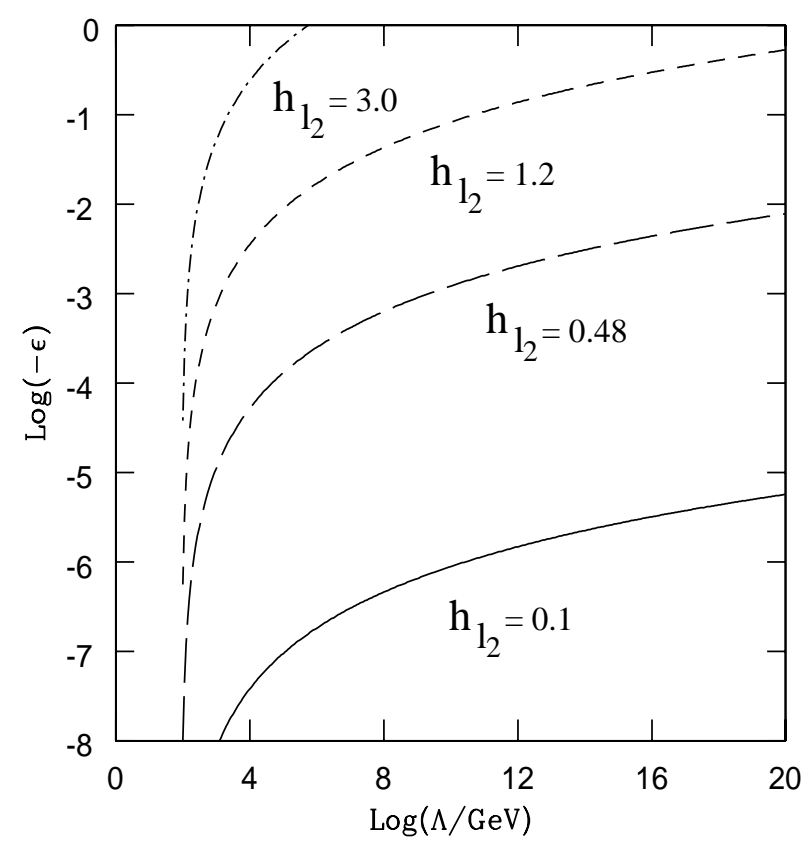

FIG. 2. Log-Log plot of $\epsilon$ vs $\Lambda$ for a few values of $h_{l_{2}} \equiv h_{\beta}$ in the MSSM.

flavor, a large mixing between flavors $\alpha$ and $\beta$ is guaranteed at the low scale if

$\epsilon_{\alpha \beta} \equiv 2\left(\delta_{\beta}-\delta_{\alpha}\right) \approx \frac{2\left(m_{\alpha \alpha}-m_{\beta \beta}\right)\left(1+\delta_{\alpha}+\delta_{\beta}\right)}{\left(m_{\alpha \alpha}+m_{\beta \beta}\right)}$,

where no summation over repeated indices is implied. Assuming that the $\Delta m^{2}$ hierarchy observed at the low scale is true at the high scale also (small radiative corrections), we have $\left|m_{1}-m_{2}\right| \ll\left|m_{2}-m_{3}\right|$. The condition for $U_{\mu 3}$ to be maximal is then

$$
\epsilon_{\mu \tau} \approx \frac{\left(m_{3}-m_{2}\right)\left(\left|U_{\tau 3}\right|^{2}-\left|U_{\tau 2}\right|^{2}\right)}{m} .
$$

In all the models in which $h_{\tau}$ dominates over $h_{e}$ and $h_{\mu}$, we have $\epsilon_{e \tau} \approx \epsilon_{\mu \tau}$. Then, the condition for the enhancement of $U_{\mu 3}$ (21) is similar to the condition for the enhancement of $U_{e 3}$ (with the replacement $[\mu \rightarrow e, 2 \rightarrow 1]$ ) if all the neutrinos have the same $C P$ parity, assuming that both $U_{\tau 1}, U_{\tau 2} \ll U_{\tau 3}$. That would imply that when $U_{\mu 3}$ is magnified due to radiative corrections, so is $U_{e 3}$. Then one cannot naturally get a small value of $U_{e 3}$ at the low scale, as is suggested by the CHOOZ data [8]. Thus, in the three generation case with quasidegenerate Majorana neutrinos, we need the $C P$ phase of one neutrino opposite to that of the other two in order for our condition to be implementable. It should be noted that satisfying this condition still does not guarantee the stability of small $U_{e 3}$.
In conclusion, we have derived a model independent condition that guarantees a large mixing at the low scale irrespective of the mixing angle at the high scale, for two quasidegenerate Majorana neutrinos with the same $C P$ parity. The condition relates the masses at the high scale to the radiative corrections. In the case of SM and MSSM, this predicts the sign of the mass difference between the neutrinos and also gives a range for its magnitude. In MSSM, it translates into a relation between the value of the high scale $\Lambda, \tan \beta$, and the common mass of the neutrinos. Extending the argument to three quasidegenerate Majorana neutrinos, we again show in a model independent way that the CP parity of one of the neutrinos should be opposite to that of the others for our conditions to be implementable at the phenomenological level.

We thank WHEPP-6, Chennai, India, where a part of the work was completed. The work of R. N. M. is supported by the NSF Grant No. PHY-9802551. The work of M. K. P. is supported by Project No. 98/37/9/BRNS-cell/731 of the Government of India.

*Electronic address: balaji@imsc.ernet.in

†Electronic address: Amol.Dighe@cern.ch

†Electronic address: rmohapat@physics.umd.edu

${ }^{\S}$ Electronic address: mparida@vsnl.com

[1] Super-Kamiokande Collaboration, Y. Fukuda et al., Phys. Rev. Lett. 82, 2644 (1999).

[2] J. N. Bahcall, P. I. Krastev, and A. Yu. Smirnov, Phys. Rev. D 58, 096016 (1998); G. L. Fogli, E. Lisi, A. Marrone, and G. Scioscia, Phys. Rev. D 59, 033001 (1999); M. C. Gonzalez-Garcia, P. C. de Holanda, C. Pena-Garay, and J. W. F. Valle, hep-ph/9906469 (to be published).

[3] T. Kajita, in Proceedings of PASCOS 99, 1999.

[4] E. Gawiser and J. Silk, Science 280, 1405 (1998); J. Primack and M. A. K. Gross, astro-ph/9810204.

[5] N. Haba, N. Okamura, and N. Sugiura, hep-ph/9810471; N. Haba, Y. Matsui, N. Okamura, and M. Sugiura, hep-ph/ 9908429.

[6] E. Ma, J. Phys. G 25, L97-L100 (1999).

[7] J. Ellis and S. Lola, Phys. Lett. B 458, 310 (1999); J. A. Casas, J. R. Espinosa, A. Ibarra, and I. Navarro, hep-ph/ 9905381.

[8] CHOOZ Collaboration, M. Apollonio et al., Phys. Lett. B 420, 397 (1998); M. Narayan, G. Rajasekaran, and S. Uma Sankar, Phys. Rev. D 58, 031301 (1998); S. M. Bilenky and C. Giunti, hep-ph/9802201.

[9] K. S. Babu, C. N. Leung, and J. Pantaleone, Phys. Lett. B 319, 191 (1993); P. H. Chankowski and Z. Pluciennik, Phys. Lett. B 316, 312 (1993).

[10] Z. Maki, M. Nakagawa, and S. Sakata, Prog. Theor. Phys. 28, 870 (1962).

[11] V. Barger, S. Geer, R. Raja, and K. Whisnant, hep-ph/ 9911524.

[12] A. S. Dighe and A. Yu. Smirnov, hep-ph/9907423. 